\title{
Evaluación de la congelación para conservación de especies autóctonas bacterianas
}

\author{
Ligia Consuelo Sánchez Leal MScㄹ, Lucía Constanza Corrales Ramírez MSc ${ }^{1}$ \\ ${ }_{1}$ Programa de Bacteriología y Laboratorio Clínico, Facultad Ciencias de la Salud, Universidad Colegio Mayor de Cundinamarca. \\ Correspondencia: Icorralesr@unicolmayor.edu.co \\ Recibido: 22-09-05 / Aceptado: 27-10-05
}

\begin{abstract}
Resumen
Los microorganismos bacterianos constituyen una buena parte de la biodiversidad de nuestro planeta y la importancia de su conocimiento y preservación se ha incrementado a medida que se descubren sus múltiples utilidades en diferentes campos. La conservación se puede hacer por diferentes métodos y uno de los que brinda mejores resultados es la congelación; por ello se realizó este proyecto cuyo objetivo fue evaluar los efectos de la congelación en 18 especies bacterianas autóctonas de la colección de cultivos del Programa de Bacteriología de la Universidad Colegio Mayor de Cundinamarca, Bogotá- Colombia. Los microorganismos incluidos en el estudio, se seleccionaron teniendo en cuenta la variabilidad de actividad metabólica entre especies y su importancia clínica, industrial, biotecnológica o ambiental. Los resultados obtenidos fueron similares en las especies analizadas y aunque en algunas se observó dificultad para recuperar su viabilidad y actividad metabólica en la primera siembra, después de esta presentaron una mejor respuesta; lo que permitió concluir que la estabilidad fenotípica y genotípica de los microorganismos estudiados, no parece depender del medio de cultivo ni del conservante utilizado, sino de los procedimientos de congelación y descongelación con los cuales se realiza el experimento. La recomendación del grupo investigador es establecer estandarizaciones y protocolos propios que garanticen la viabilidad y estabilidad bioquímica de los microorganismos que se conserven por este método.
\end{abstract}

Palabras clave: congelación, descongelación, viabilidad bacteriana, actividad metabólica.

\begin{abstract}
The bacterial microorganisms constitute a good part of the biodiversity of our planet and the importance of its knowledge and preservation has been increased as their multiple utilities in different fields are discovered. The conservation can be done by different methods and one of which offers better results is the freezing; for that reason east project was made whose objective was to evaluate the effects of the freezing in 18 native bacterial species of the collection of cultures of the Program of Bacteriology of the University Colegio Mayor de Cundinamarca, Bogotá-Colombia. The microorganisms including in the study, they were selected considering the variability of metabolic activity between species and their clinical importance, industrialist, biotechnological or environmental. The obtained results were similar in the analyzed species and although some had difficulty to recover their viability and metabolic activity in the first sowing, later they presented displayed one better answer, what allowed to conclude that the phenotypic and genotypic stability does not seem to depend on
\end{abstract}


means of culture nor on the used preservation substance, but of the procedures of freezing and defrost with which the experiment was made. The recommendation of the investigating group is to establish own standardizations and protocols that guarantee the viability and biochemical stability of the microorganisms that are conserved by this method.

Key words: freezing, defrost, viability, metabolic activity.

\section{Introducción}

El mundo microscópico que conforma la naturaleza y que rodea al ser humano es un microcosmos que poco a poco se ha ido explorando y que ha llevado a planteamientos nuevos sobre las relaciones que se establecen entre organismos microscópicos vivos y el hombre; lo que fue en un primer momento visto como un problema, se ha constituído en un excelente recurso para desarrollar nuevas tecnologías. Esta nueva relación obliga al hombre a tomar medidas para tener un conocimiento más profundo de ellos, a pensar en su preservación y a mejorar los métodos para su conservación. Es por ello que la conservación de la biodiversidad microbiana autóctona se debe dirigir a mantener viables y estables fenotípica y genotípicamente especimenes representativos con aplicabilidades concretas, para lo cual es necesario desarrollar técnicas de conservación específicas para cada una de ellas previendo la reducción de efectos adversos en el proceso.

El Programa de Bacteriología y Laboratorio Clínico de la Universidad Colegio Mayor de Cundinamarca, Bogotá- Colombia, desde hace más de 20 años posee una diversidad de microorganismos bacterianos, los cuales han sido conservados con almacenamientos a corto plazo (congelación a $4^{\circ} \mathrm{C}$ ), con pases continuos en el caso de microorganismos bacterianos de difícil recuperación y a mediano plazo utilizando Agar Base Sangre $B A B$ en tubo recto a temperatura ambiente con resultados satisfactorios. El mayor problema de estos métodos son las resiembras porque ello constituye una posibilidad de cambio en su comportamiento tanto genotípico como fenotípico y por lo tanto, si bien estos microorganismos conservan sus características por un tiempo, no se tendría certeza de que el microorganismo haya tenido cambios transitorios o permanentes.

Esta observación llevo a valorar otros métodos que sean más reproducibles y confiables con el fin de garantizar la calidad del microorganismo conservado y constituir una oferta de servicios competitiva a la altura de instituciones nacionales e internacionales. Entre estos métodos se consideran la liofilización y congelación a diferentes temperaturas como los más utilizados. El análisis llevó a escoger la congelación para la conservación por las ventajas ofrecidas: facilidad para procesar los microorganismos, confiabilidad de los resultados, estabilidad de las cepas de microorganismos y el costo. Al respecto, Ann Tanghe y Frederick Dumont $(1,2)$ hacen una revisión de los determinantes de tolerancia a la congelación y concluyen que los microorganismos han desarrollado mecanismos de adaptación al ambiente de estrés que genera la congelación. Un factor que se debe considerar en este método es el relacionado con las sustancias que brindan protección a los posibles efectos dañinos causados por las bajas temperaturas como la formación de cristales de hielo intracelular. Han Bumsoo y Jason Acker $(3,4,5)$ establecen que el mecanismo de daño celular por congelación más significativo es la formación de cristales y que es necesario entender dicho mecanismo para prevenirlo es así, como se han considerado como protectores diversas sustancias que son efectivas y que evitan la formación de estos cristales. Hubálek y Arlet de Saab (6-9) presentan una revisión sobre las sustancias criopreservantes más utilizadas, sus ventajas y limitaciones, producto del análisis de estas, se optó por 


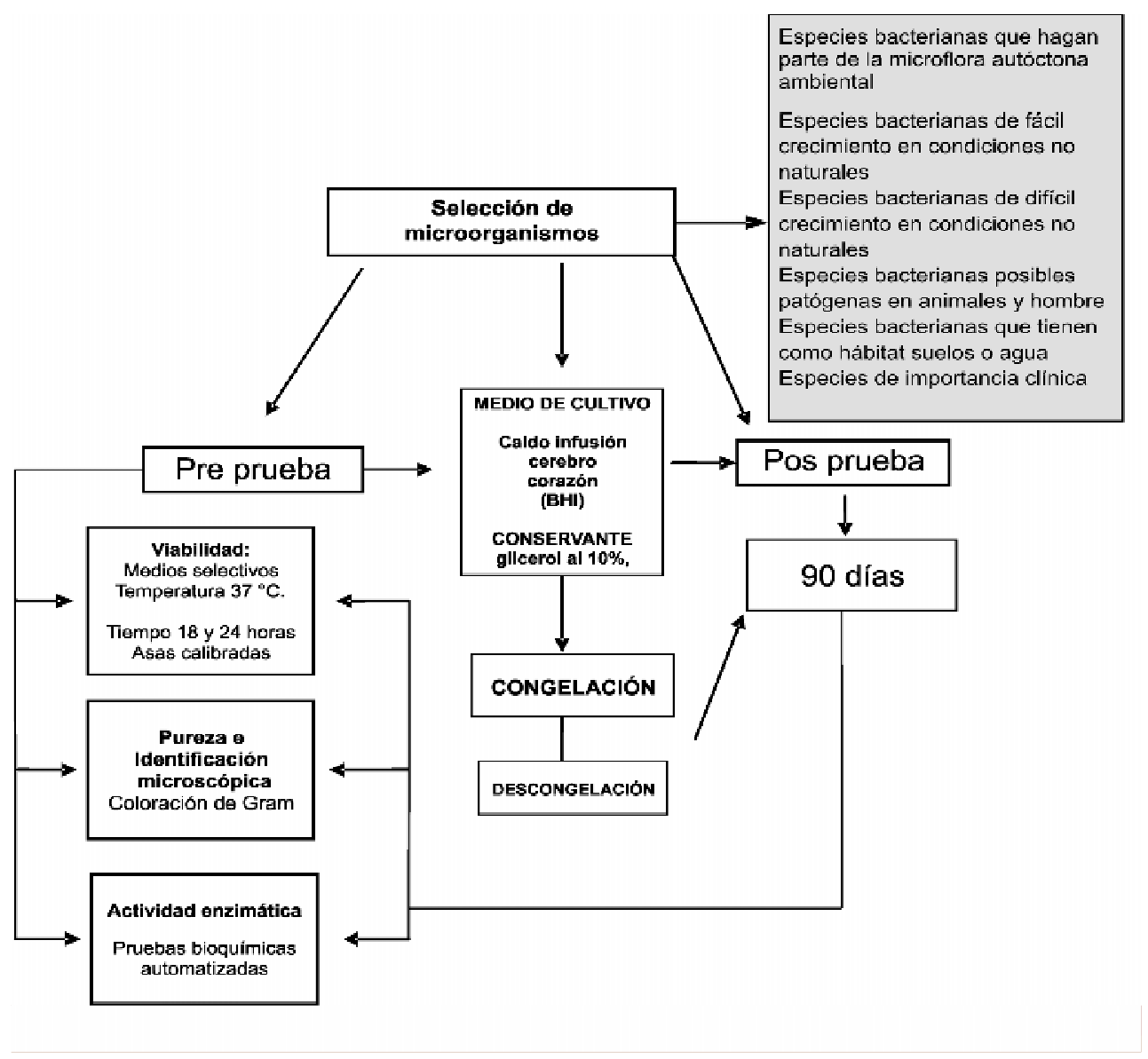

Figura 1. Protocolo para la evaluación de la congelación para conservación de especies autóctonas bacterianas

utilizar el glicerol. Otro aspecto considerado fue el relacionado con la diversidad en el comportamiento metabólico, auxotrofismo de los microorganismos seleccionados para el estudio, el cual llevó a establecer una normatividad o estandarización por género y en algunos casos por especie que garantizara el éxito en el proceso congelación - descongelación.

Esta investigación constituyó la primera fase del proyecto "Métodos óptimos de conservación de la microdiversidad autóctona”. El objetivo de esta fase fue evaluar el método de congelación como método de conservación de la microdiversidad autóctona en 18 especies bacterianas, indispensables para el desarrollo de procesos académicos, de investigación y de extensión los cuales formarán parte de la Colección de Cultivos del Programa de Bacteriología de la Universidad Colegio Mayor de Cundinamarca.

\section{Materiales y métodos}

Selección de microorganismos:

Los criterios para la selección fueron:

1. Especies bacterianas que conforman la microflora autóctona ambiental.

2. Especies bacterianas de fácil crecimiento en condiciones no naturales (ex situ).

3. Especies bacterianas de difícil crecimiento en condiciones no naturales (ex situ)

4. Especies bacterianas que tienen como hábitat suelos o agua y se consideran posibles patógenos para los seres humanos.

5. Especies bacterianas de importancia clínica: (i) posibles patógenos en humanos y animales, (ii) de importancia industrial en el control de calidad en procesamiento de alimentos, (iii) preservación de su hábitat natural, (IV) de importancia 
biotecnológica utilizados como sustratos de utilización en diversos procesos.

No se utilizaron microorganismos bacterianos anaerobios estrictos.

Los microorganismos seleccionados fueron: Staphylococcus aureus, Staphylococcus saprophyticus, Streptococcus pyogenes, Streptococcus agalactiae, Streptococcus pneumoniae, Enterococcus faecalis, Lysteria monocytogenes, Klebsiella oxytoca, Proteus mirabilis, Salmonella typhi, Pseudomona fluorescens, Morganella morganii, Citrobacter freundii, Shigella flexnerii, Escherichia coli, Vibrio cholerae, Acinetobacter boumanii y Acinetobacter calcoaceticus.

\section{Temperatura de congelación:}

El método elegido para la conservación de los microorganismos fue $-20^{\circ} \mathrm{C}$.

\section{Medios de cultivo:}

Se utilizó caldo infusión cerebro corazón (BHI) preparado de acuerdo con las instrucciones de la casa comercial (Difco). Se llevo a esterilizar en autoclave horizontal a 121 libras de presión por 15 minutos y se almacenaron en crioviales con capacidad de $1.2 \mathrm{mls}$.

\section{Conservante:}

Se utilizó glicerol al 10\%, el cual fue añadido a cada tubo, una vez sembrada la suspensión de células bacterianas a congelar.

\section{Desarrollo de pretratamientos:}

Viabilidad: los microorganismos fueron inoculados en los medios selectivos para su crecimiento. Se incubaron a la temperatura óptima de cada uno entre 18 y 24 horas y se observó su crecimiento. La siembra se realizó con asas calibradas para garantizar el mismo inóculo.
Pureza e Identificación microscópica mediante coloración de Gram: se observó el crecimiento en los medios selectivos y se comprobó si las colonias presentaban homogeneidad. Se procedió a realizar una coloración de Gram de 3 colonias diferentes para comprobar la compatibilidad morfológica con el microorganismo esperado.

Actividad enzimática: se realizaron montajes de pruebas bioquímicas automatizadas a partir del crecimiento obtenido a las 18 horas en los medios selectivos, siguiendo los procedimientos establecidos para ello. A las 18 horas se aplicaron los reactivos solicitados por el sistema y se procedió a su lectura.

\section{Preparación de microorganismos a congelar}

Una vez confirmada la viabilidad, pureza, identificación microscópica por coloración de Gram y actividad enzimática de los microorganismos, se procedió a preparar suspensiones de bacterias con $7.5 \mathrm{x}$ $10^{8} \mathrm{UFC} / \mathrm{ml}$, Escala de Mc Farland 4, posteriormente se colocó en cada vial de medio BHI, $100 \mu 1$ de bacterias en suspensión y se agregó el glicerol al 10\% (100 $\mu 1$ por $1 \mathrm{ml}$ de medio) y se llevo a congelar en forma inmediata a $-20^{\circ} \mathrm{C}$.

\section{Descongelación}

El procedimiento para la descongelación de los microorganismos en estudio fue:

1. Se retiró del vial congelado a $-20^{\circ} \mathrm{C}$ y verificó que estuviera bien cerrado.

2. Se espero hasta la descongelación completa del vial antes de realizar la recuperación de los microorganismos.

3. El medio de cultivo líquido elegido para reactivar los microorganismos fue caldo BHI, preparado en tubos de 13 x 100 en cantidad de $3 \mathrm{ml}$; se preparó 5 días antes y se conservó entre 2 y $8{ }^{\circ} \mathrm{C}$.

4. Se sembró una asada de cada microorganismo con asa calibrada estéril y se inoculo en el BHI a $37^{\circ} \mathrm{C}$ por tres (3) horas, antes de ser sembrados en los medios selectivos. 
5. Los viales con los microorganismos fueron también colocados a $37^{\circ} \mathrm{C}$ por tres horas para realizar una siembra directa en los medios selectivos.

6. Los medios de cultivo selectivos elegidos para la recuperación de los 18 microorganismos fueron: agar sangre, agar Mac. conkey y agar nutritivo con $\mathrm{NaCl}$ al 1\% (Vibrio cholerae). Los cuales se ambientaron por 15 minutos a $37^{\circ} \mathrm{C}$ antes de ser sembrados.

7. A las tres (3) horas, se sembraron en los medios selectivos los microorganismos de los viales descongelados y del BHI ambientados y se incubaron a $37^{\circ} \mathrm{C}$ por 18 horas.

8. La siembra se realizó por doble estría. Se utilizó la misma caja 50/50 con división para verificar crecimiento y viabilidad desde el BHI y el vial descongelado.

9. Los medios selectivos inoculados se colocaron a $37^{\circ} \mathrm{C}$ por 24 horas .

10. Se realizó lectura de las siembras a las 24 y 48 horas para observar viabilidad

\section{Realización de Post pruebas}

Se confirmó viabilidad, pureza, identificación microscópica por coloración de Gram y actividad enzimática en los 90 días a partir del momento en que se congelaron los microorganismos.

\section{Resultados}

Ver Tabla 1. Resultados, en la página siguiente.

\section{Preprueba}

Los 18 microorganismos utilizados como muestra fueron identificados plenamente en el laboratorio por crecimiento en medios selectivos, coloración de Gram y pruebas bioquímicas automatizadas, las cuales reportaron entre un 98 y $99 \%$ de confiabilidad.

El origen de estos microorganismos está consignado de acuerdo con el Protocolo de depósito del sistema de colección de cultivos del Programa de Bac- teriología de la Universidad Colegio Mayor de Cundinamarca en la ficha de cada microorganismo.

\section{Posprueba}

\section{Viabilidad}

Al observar los medios de cultivo selectivos (agar sangre, agar Mac Conkey y Agar nutritivo con $\mathrm{NaCl}$ al 1\% (Vibrio cholerae) en los que se sembraron los microorganismos congelados, se evidenció poco crecimiento, entre 5 y 20 UFC. Sin embargo, el crecimiento en el medio líquido caldo BHI evidenció un crecimiento mayor a $10^{8}$ UFC a las 24 horas de incubación. Por esta razón se tomó la totalidad de los caldos BHI y los microorganismos se sembraron nuevamente en medios selectivos específicos, se incubaron por 24 horas a $37^{\circ} \mathrm{C}$ y se observó nuevamente la viabilidad. El crecimiento estuvo entre 60 y 100 UFC en todos los casos con esta nueva siembra, lo cual permite evidenciar que los microorganismos requieren un medio que les permita recuperar su actividad enzimática en forma adecuada.

Los resultados obtenidos con S. pneumoniae demuestran que probablemente el método de congelación a $-20^{\circ} \mathrm{C}$ no es el más adecuado por cuanto no se observa crecimiento en el cultivo directo del vial congelado, ni en el preincubado por 3 horas en caldo BHI y el crecimiento obtenido en el caldo BHI por 18 horas mostró crecimiento mixto en donde no fue posible recuperar el microorganismo $(10,11)$.

\section{Pureza}

No hubo contaminación de los microorganismos con otras especies, ni géneros. La homogeneidad de las colonias y la identificación microscópica con tinción de Gram permitieron realizar esta confirmación. Sin embargo, se pudo evidenciar que algunos bacilos Gram negativos presentaron cambios estructurales al ser observados al microscopio como fue el caso de la Klebsiella oxytoca que presentó acomodación en empalizada no característica. 


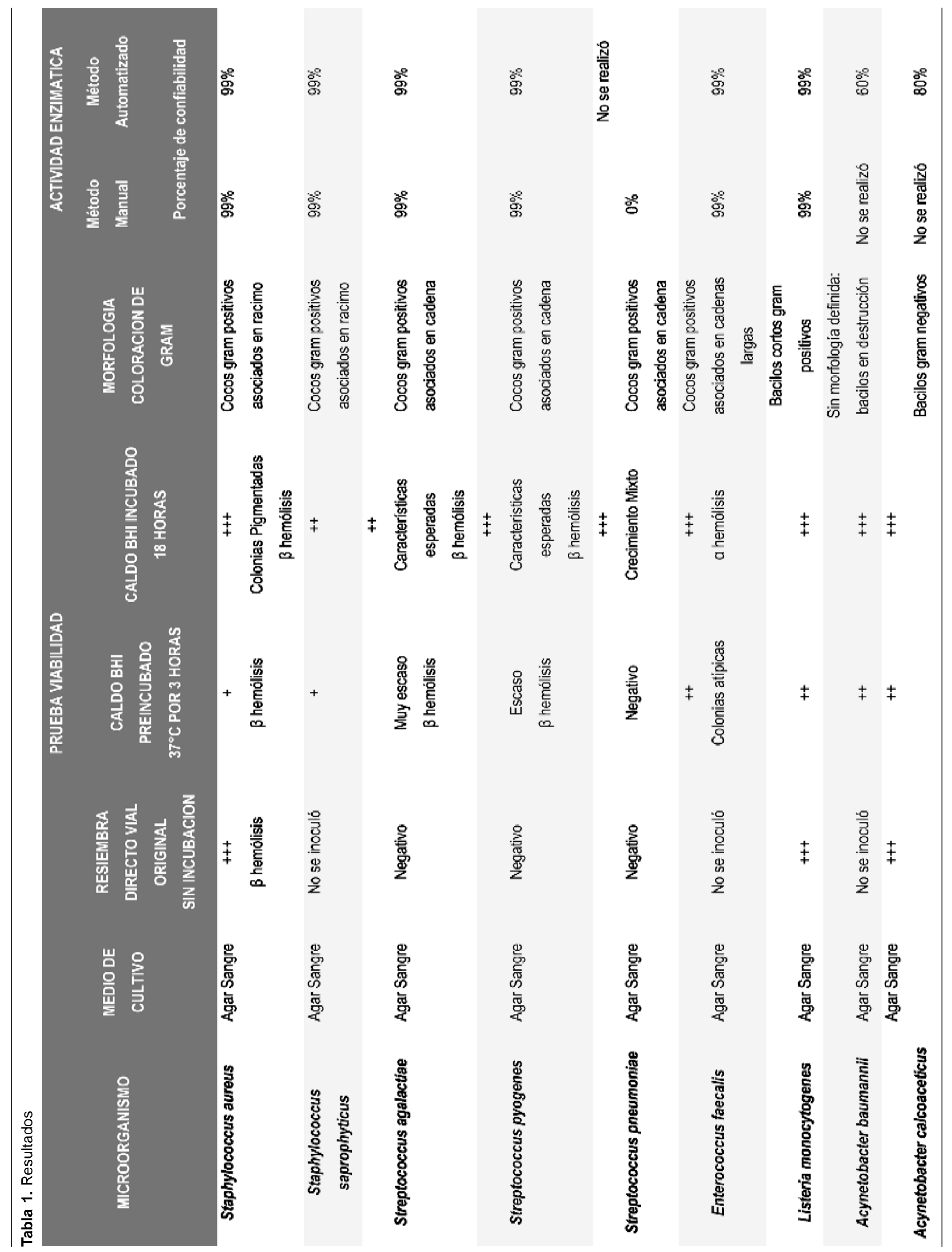




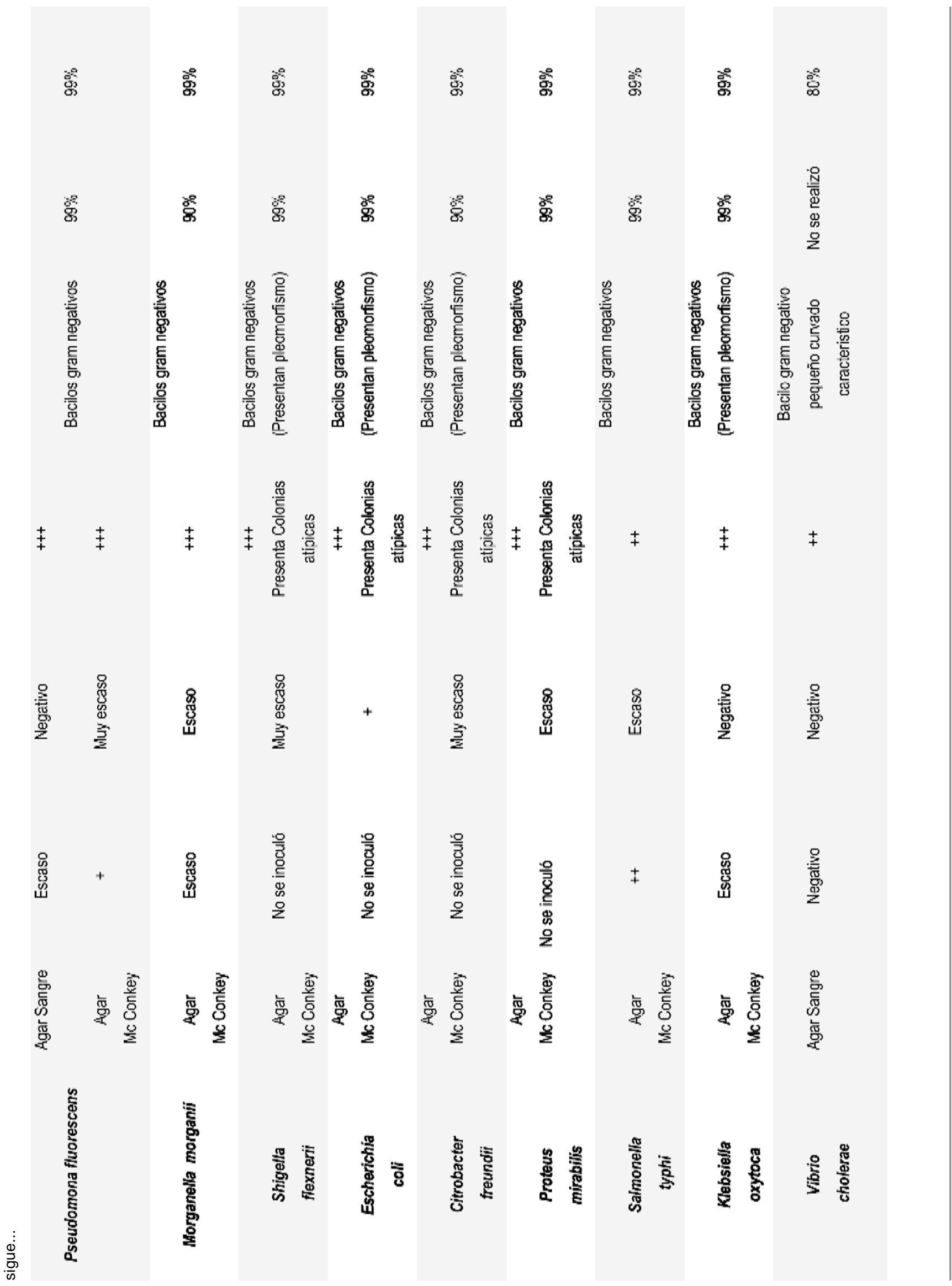




\section{Actividad enzimática}

Los resultados obtenidos por pruebas automatizadas con las bacterias Gram positivas reportaron una confiabilidad de 90 al 99\%. Sin embargo, algunas bacterias Gram negativas como Citrobacter freundii, Morganella Morganii y Shigella flexnerii evidenciaron cambios en algunas de sus reacciones bioquímicas características, las cuales fueron recuperadas después de dos resiembras más en sus medios selectivos.

\section{Discusión}

La congelación es un proceso físico químico que genera estrés a las células vivas pero que se convierte en una alternativa útil para la conservación de microorganismos bacterianos.

Los resultados obtenidos permiten confirmar que la actividad bioquímica de la bacteria se ve afectada por el estrés, pero que es un estado transitorio. Cuando el microorganismo se estabiliza y se elimina la causa de estrés, los procesos moleculares son recuperados en su totalidad. Sin embargo, el estrés producido por el procedimiento de congelación debe ser mitigado en el momento del procedimiento de descongelación y el medio que se provea a los microorganismos bacterianos debe ser líquido para que facilite el intercambio molecular iónico y se restituya la actividad bioquímica de la membrana rápidamente.

Se evidencio que el medio líquido utilizado, caldo BHI, facilitó más la recuperación del equilibrio iónico de la membrana y por lo tanto la producción de ATP para reestablecer su actividad bioquímica. El estrés parece jugar un papel importante en la recuperación de estas características y varios investigadores se han referido a este tema. Por esta razón, es esencial considerar dentro del protocolo de descongelación un paso inicial de acomodación o recuperación de la actividad bioquímica que disminuya al máximo el estrés en el que se ha encontrado por la congelación. (8). Algunos autores incluso han llegado a referirse en términos de resucitación, cuando el microorganismo vuel- ve a recuperarse después de un proceso de congelación (8). Así mismo, se ha planteado como las bacterias están capacitadas con una maquinaria biomolecular que incluye proteínas anti congelantes $(5,9)$ y se ha establecido que los microorganismos en congelación o en estado de "hambre", starvatión survival - SS, conservan una alta actividad metabólica para soportar cambios metabólicos y activar mecanismos de reparación, relacionados con el mantenimiento de la integridad celular y de esta forma con la capacidad de ser cultivable (8).

La protección de estas células vivas bacterianas con un crioprotector es necesaria. El glicerol ha demostrado ser una de las mejores sustancias por cuanto sus características moleculares le permiten simular una vitrificación alrededor de la bacteria, lo cual impide que la formación de cristales de hielo lesione las membranas citoplasmáticas.

Se pudo evidenciar que algunos bacilos Gramnegativos presentaron cambios estructurales al ser observados al microscopio como fue el caso de la Klebsiella oxytoca que presento acomodación en empalizada no característica. Este resultado hace pensar que la hipótesis planteada sobre el estrés por congelación para algunos microorganismos constituye una respuesta más evidente que para otros. Es así, como las bacterias Gram negativas parecen presentar más cambios moleculares de adecuación estructural en condiciones de estrés por congelamiento que las bacterias Gram positivas. Este fenómeno esta relacionado con la conformación química de su membrana y por otro tipo de mensajes o señalizaciones, quórum sensing (8).

\section{Referencias}

1. Tangue P, Van D, Thevelein J. Determinants of freeze tolerance in microorganisms, physiological importance and biotechnological applications. Advances in applied Microbiology 2003;53:129 - 176.

2. Dumont F, Marechal P, Gervais P. Influence of cooling rateo in Saccharomyces cerevisiae destruction during freezing: unexpected viability at ultra-rapid cooling rates. Criobiology 2003; 46: 33-42.

3. Ham B, Bischof J. Direct cell injury associated with eutectic crystallyzation during freezing. Criobiology 2004; 48: 8-21.

4. Wowk B, Fahy G. Inhibition of bacterial ice nucleation by polyglicerol polymers Volume. Criobiology 2002; 44:14-23. 
5. Acker J, Locksley E. Protective effect of intracellular ice during freezing. Criobiology 2003; 46: 197-202.

6. Hubalek Z. Review. Protectants used in the cryopreservation of microorganisms. Criobiology 2003; 46: 205-229.

7. Aulet De Saab O. y otros. Comparative Study of Preservation and Storage of Haemophilus influenzae Memórias do Instituto Oswaldo Cruz 2001;96(4).

8. Mukamolova G, Kaprelyants A, Kell D, Young M. Adoption of the transiently non-culturable state a bacterial survival strategy. Advances in applied Microbiology 2003;47:65 - 129.

9. Wang Jung Hui. A comprehensive evaluation of the effects and mechanisms of antifreeze proteins during low temperature preservation. Criobiology 2000; 41: 1 - 9 .

10. Charalambous B. M., y colaboradores. Quantitative Validation of Media for Transportation and Storage of Streptococcus pneumoniae. Journal of Clinical Microbiology 2003; 41(12):5551-5556.

11. Siberry George, Brahmadathan K.N. et al. Comparison of different culture media and storage temperatures for the longterm preservation of Streptococcus pneumoniae in the tropics. Bulletin of the world health organization 2001; 79: $43-47$. 